\title{
CÁLCULOS DE CONSUMO E EMISSÕES EM VEÍCULOS HÍBRIDOS PLUG-IN EM REDUÇÃO DE CARGA DA BATERIA
}

\author{
Gabriel Murgel Branco ${ }^{1}$ \\ Fábio Cardinale Branco ${ }^{1}$ \\ Edson Orikassa ${ }^{2}$ \\ Sylvia Talita Ferreira ${ }^{2}$
}

Os veículos híbridos "plug-in" representam um avanço tecnológico que permite a utilização de um veículo tanto com a energia elétrica obtida da rede doméstica de distribuição quanto com um combustível consumido ou, ainda, uma combinação das duas fontes de energia.

Por um lado, esta versatilidade permite que o usuário escolha a opção que lhe seja mais conveniente em função das distâncias que pretende percorrer, mas por outro, é preciso oferecer-lhe os parâmetros adequados para que faça uma análise da eficiência energética resultante das suas opções em cada caso.

Este trabalho apresenta três formas de interpretação dos resultados de ensaios de emissões de poluentes e dos consumos de combustível e energia elétrica, para orientação dos usuários em seus modos particulares de uso, ou para padronizá-los para a certificação e comparação tecnológica de forma independente do usuário, ou ainda para estimar os resultados de uma frota para planejamento e formulação de políticas públicas. Neste último aspecto, este trabalho propõe um ajuste da norma internacional para os hábitos de utilização de veículos nas cidades brasileiras, a partir das estatísticas levantadas através do Programa de Inspeção e Manutenção de São Paulo - I/M-SP.

\section{FUEL CONSUMPTION AND EMISSIONS CALCULATIONS IN PLUG-IN HYBRID VEHICLES DURING BATTERY CHARGE DEPLETING MODE}

Hybrid vehicles "plug-in" represent a technological breakthrough that allows the use of a vehicle with electric energy obtained from domestic distribution network and a consumable fuel, or a combination of the two energy sources.

On the one hand, this versatility allows the user to choose the most convenient option depending on the distances he want to go, but on the other, it is necessary to provide him with the appropriate parameters to make an analysis of energy efficiency resulting from each option.

This paper presents three approaches to interpret test results of pollutant emissions and fuel and electric energy consumption, to guide users in their particular modes of use, or for standardyzing them for certification and technological comparison independently of the user option, or even to estimate the results of a fleet average and formulation of public policies planning. In this last aspect, this paper proposes an standard for vehicle use habits in Brazilian cities, adapted from the international standards, which is derived from the statistics raised through Inspection and Maintenance Program in São Paulo I/M-SP.

\footnotetext{
${ }^{1}$ EnvironMentality - Consultor

${ }^{2}$ Toyota do Brasil
} 


\section{CÁLCULOS DE CONSUMO E EMISSÕES EM VEÍCULOS HÍBRIDOS PLUG-IN EM REGIME DE REDUÇÃO DE CARGA DA BATERIA}

O comportamento de um veículo elétrico híbrido recarregável (plug-in) enquanto a bateria se descarrega é altamente variável em termos de emissões e dos consumos de energia elétrica e de combustível, em decorrência da redução do estado de carga da bateria ${ }^{3}$.

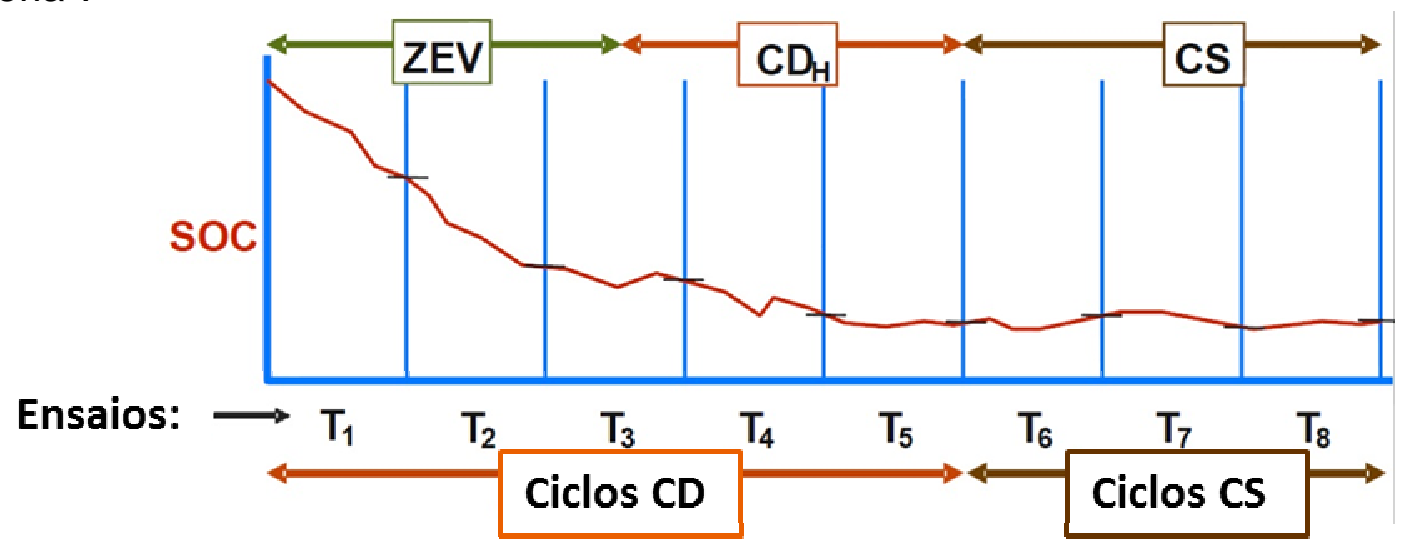

Figura 1 - Estado de carga ao longo de vários ciclos de condução (PriusHymotion)

Por isso, o ensaio do veículo com a bateria totalmente carregada deve ser composto de ciclos de condução sucessivos, tantos quanto forem necessários até que o estado de carga da bateria se estabilize na faixa em que o veículo se comporta como um híbrido normal. Neste estado, o movimento é assegurado somente pela energia proveniente do combustível e a bateria restringe-se apenas ao equilíbrio dos picos de demanda e regeneração de energia.

Os resultados desses ensaios sucessivos devem ser organizados numa tabela $e$ podem ser interpretados de várias maneiras, dependendo do interesse ser voltado ao usuário ou à definição de políticas públicas.

\section{Interesse do usuário}

A escolha de um veículo depende da adequação das suas características aos hábitos do usuário, especialmente à extensão do seu trajeto diário. Para uma avaliação específica sob o ponto de vista do usuário, as médias acumuladasdesde a partida do primeiro ciclo oferecem os parâmetros de maior interesse, ou seja, os que permitem uma estimativa dos gastos em energia elétrica e em combustível, em função dos hábitos do usuário.

Neste caso e assumindo-se uma recarga da bateria por dia, os ciclos de condução devem ser realizados progressivamente e o cálculo do consumo e das emissões deve ser realizado separadamente para cada um.Portanto, para atender às

\footnotetext{
${ }^{3} \mathrm{O}$ termo bateria aqui é usado em lugar de "sistemas de armazenagem de energia elétrica" por ser o mais comum nos veículos recarregáveis, entretanto existem outros conceitos tais como ultracapacitores, volantes e sistemas hidropneumáticos que podem desempenhar a mesma função.
} 
necessidades do usuário, estes parâmetros - especialmente o consumo - devem ser calculados em função da distância percorrida diariamente (entre duas recargas da bateria)e deverão ser apresentados como uma série de resultados acumulados de, digamos, 1 a 15ciclos urbanos (correspondentes a $180 \mathrm{~km} / \mathrm{dia}$, que representa os veículos de uso intenso, como taxis), considerando os "n" ciclos da série com redução de carga da bateria e os demais com os resultados obtidos no ciclo seguinte com sustentação de carga, conforme equações a seguir:

$\operatorname{Yacum}_{n}($ para $n$ ciclos, em gramas ou litros ou $W h / k m)=\frac{\sum_{1}^{m} Y_{i}(\text { gramas ou litros })}{\sum_{1}^{n} D_{i}(\mathrm{~km})}$

Onde:

$n$ = número de ciclos necessários para a estabilização da bateria;

$m \quad=$ número de ciclos considerados para cada valor médio acumulado. Deve ser assumido de 1 a 15 para a construção de uma série a ser apresentada como uma curva Yacum $_{m}=f(m)$;

$i=$ número de ordem dos ciclos considerados em cada cálculo de Yacum $_{m}$;

Yacum $_{m}($ gramas ou litros ou $W h / \mathrm{km})=$ média da massa de poluente ou do volume de combustível por km, acumulados desde a partida até o "io”" ciclo considerado;

$Y_{i}($ gramas ou litros ou $W h)=$ massa de poluente, ou o volume de combustível, ou o consumo de energia AC, medidos em cada ciclo;

Para $i>n$, utilizar $Y_{i}=Y_{C S}$; no caso de energia elétrica $E_{i}=E_{C S}=0$

$Y_{C S}($ gramas ou litros ou $W h)=$ massa de poluente, ou o volume de combustível, ou o consumo de energia AC, medidos em carga sustentada após o ciclo "n".

$D_{i}(\mathrm{~km})=$ distância percorrida em cada ciclo; $(12 \mathrm{~km}$ no caso do ciclo urbano FTP 75 e 16,5km no ciclo estrada Hwy)

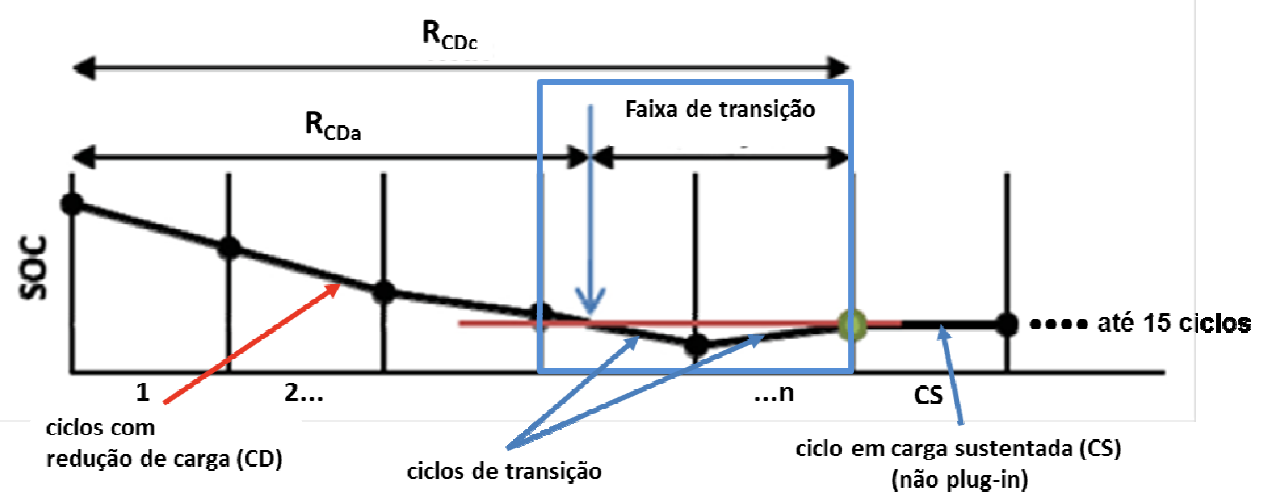

Figura 2 - Sequencia de ensaios com redução de carga da bateria 
Com esta abordagem, o fabricante terá a possibilidade de oferecer ao usuário uma curva que lhe permita estimar os consumos de combustível e de energia elétrica do veículo plug-in nas suas condições de utilização em particular. A figura 3 apresenta o consumo de combustível medido em cada ciclo urbano (padrão de $12 \mathrm{~km}$ ) e a média acumulada nos ciclos desde o primeiro. Também pode ser de interesse a combinação dos consumos de combustível e de energia elétrica em unidades equivalentes, como indicado na curva tracejada nesta mesma figura. Todos os cálculos devem ser realizados na resolução de um ciclo, pois a sua representatividade do uso normal está associada a todos os movimentos que o compõe.

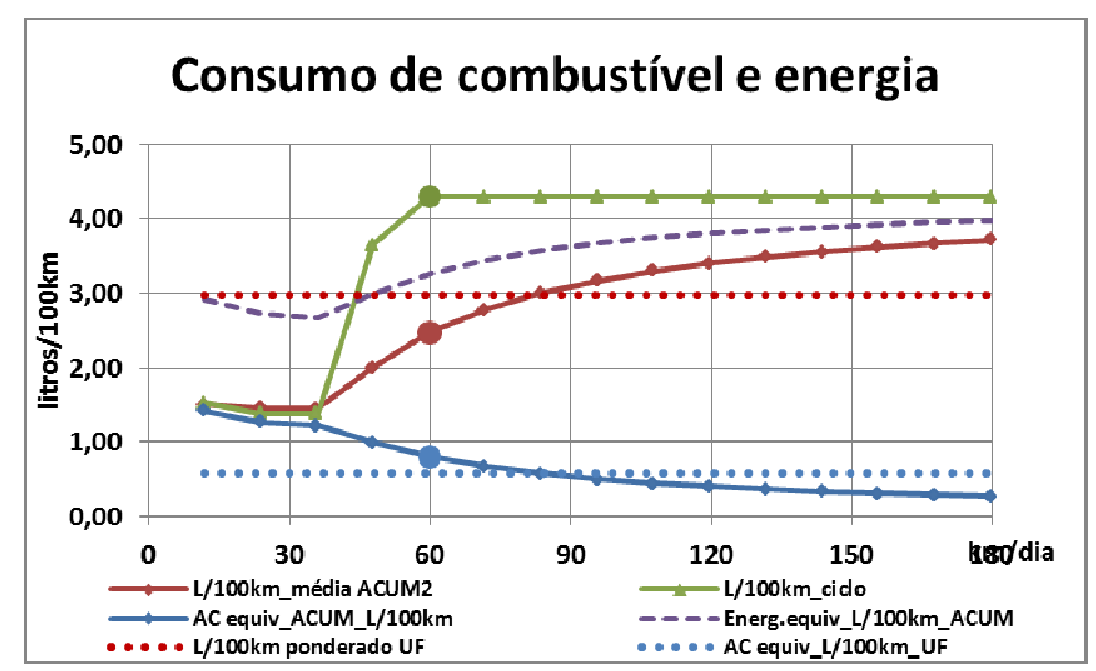

Figura 3 - Consumo de combustível e energia a cada ciclo urbano (12km)

\section{Padronização para certificação e comparação de veículos}

De uma forma mais simples, para efeito de uma comparação tecnicamente padronizada entre modelos de veículos, é recomendável padronizar as médias acumuladas numa médiade utilização "mais independente do indivíduo", isto é, associada à média estatística da população, de forma a resumi-las em duas situações:

a) médiasdurante a descarga da bateria até o ciclo "n", iniciando-se com a bateria totalmente carregada (pontos vermelho e azul ressaltados na curva da figura 3);

b) médiasdos resultados em carga sustentada (ponto verde ressaltado na figura 3), realizando um ensaio com partida a frio e outro a quente, ambos ponderados como nos veículos convencionais. Neste caso, o précondicionamento do veículo deve assegurar que a carga da bateria seja a mesma no início e no final do ciclo, para evitar distorções nos resultados por influência decontribuições líquidas (positivas ou negativas) de energia da bateria durante o ensaio.

Neste caso, os cálculos são aprimorados para que o ponto final do regime de descarga seja determinado $\left(R_{\mathrm{CDa}}\right)$ e os parâmetros sejam interpolados linearmente, conforme definido no Anexo B da norma SAE J1711. A EPA - Agência de Proteção Ambiental norteamericana - exige 0 atendimento aos limites de emissão nos dois 
casos independentemente, para a certificação de emissões.Nas etiquetas americanas para divulgação da eficiência energética, as médias de consumo de combustível são apresentadas para uso urbano, de estrada e ponderadas.

\section{Estratégias e políticas públicas}

As estratégias e políticas públicas para aumento da eficiência energética, uso de energia limpa e redução de emissões visam os hábitos da sociedade em geral ese baseiam em dados estatísticos comportamentais da população, obtidos da própria frota circulante. Tais dados, entre eles a quilometragem anual praticada são utilizados em "inventários" de emissão de poluentes e das demandas de insumos energéticos. Estes mesmos conceitos são utilizados para definir os padrões médios característicos para os cálculos dos resultados de consumo e emissões "de referência", que também podem ser utilizados para a certificação e a etiquetagem de veículos.

Os regulamentos norteamericanos de 2011 estabelecem metas utilizando a SAE J2841, que define os parâmetros estatísticos representativos do comportamento da sociedade americana em nível nacional e propõe uma padronização que é adotada nas demais normas SAE.

Aplicando-se este conceito, os resultados dos " $n$ " ensaios até a estabilização da bateria são combinados com os do ensaio em carga estabilizada e produzem um número que, associado ao "fator de utilização" com propulsão elétrica, que é função da quilometragem média diária obtida através dos dados da frota circulante, produz os resultados "médios" esperados, que se prestam à avaliação de impactos ambientais, à definição de políticas e estratégias públicas e sócio-econômicas.

\section{Fatores de Utilização e Cálculo Estatístico}

Conceitualmente, a variabilidade da quilometragem anual é muito grande, de forma que a definição do uso de energia elétrica no veículo híbrido é feita em termos de distribuição acumulada de frequênciasda oportunidade dos usuários atenderem suas necessidades de transporte enquanto a bateria ainda possui carga disponível, chamada de "fator de utilização" ou de "utilidade" da propulsão elétrica proveniente da recarga da bateria com energia externa.

A figura 4 mostra o comportamento de 5 veículos cujo alcance ou "utilidade" da sua propulsão por energia elétrica de recarga externa da bateria seja limitado, por exemplo, a $40 \mathrm{~km}$, quando o veículo passa a funcionar como um híbrido normal em carga sustentada. Além desse limite, a energia consumida será obtida totalmente do combustível, embora haja oscilação da carga da bateria em função das regenerações de energia de frenagem e demandas nas acelerações. Os dois primeiros veículos foram utilizados sempre com consumo de energia de recarga da bateria, enquanto que nos demais esta ajuda ficou limitada à proporção de $40 \mathrm{~km}$ sobre o respectivo total diário.

O Fator de Utilização (ou de utilidade) da energia de recarga, pode ser definido como a fração obtida pela divisão da distância percorrida com apoio da energia elétrica pela distância total, resultando neste exemplo em73\% da utilização dos veículos em redução de carga e $27 \%$ movido unicamente pelo combustível. 


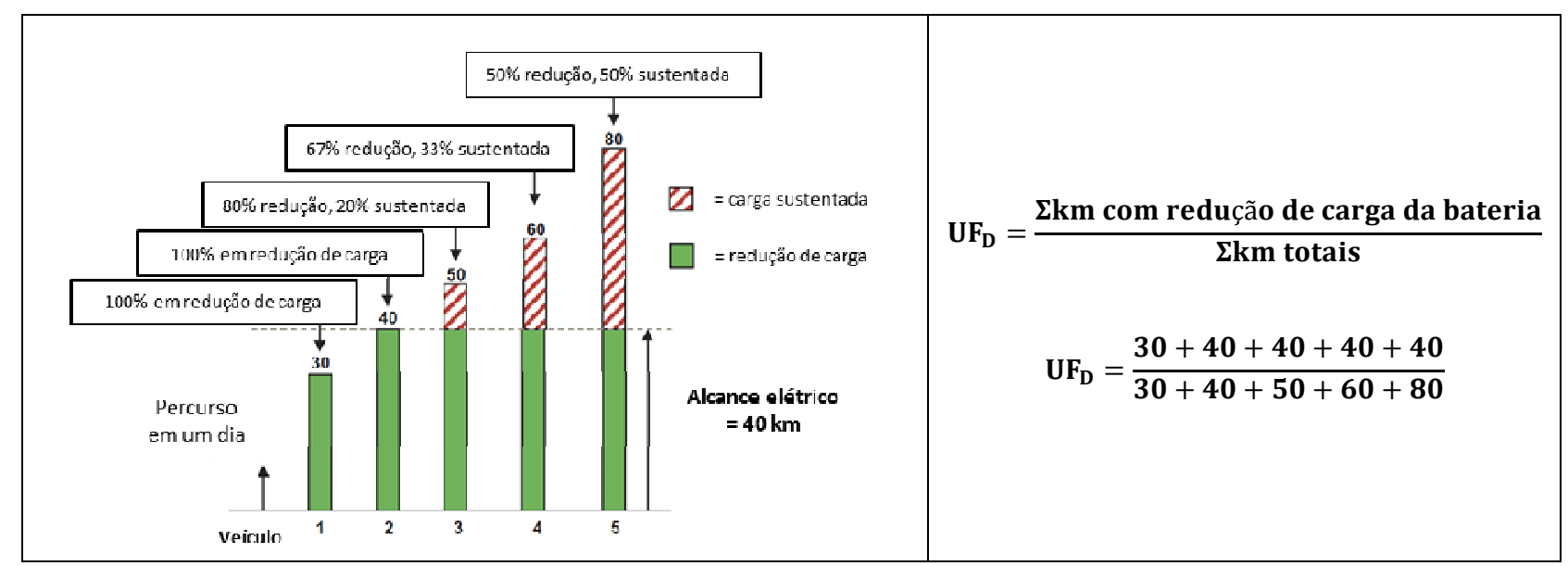

Figura 4 - Interdependência do "alcance elétrico" e a estatística de uso

Este conceito, aplicado à frota leva à definição do Fator de Utilização da Frota FUF como a proporção de uso da bateria para cumprir o percurso médio diário dos veículos da frota de interesse, em regime de redução de carga da bateria.A figura 5 esquematiza uma sequência de ensaios de um veículo percorrendo ciclos de condução padronizados (urbano ou estrada), sucessivamente desde a carga total da bateria até a sua estabilização. A representatividade dos ciclos quanto ao tráfego normal é assegurada pelo ciclo completo, de forma que a resolução das medições é de 1 ciclo, não sendo adequado interromper as medições antes dos finais dos mesmos

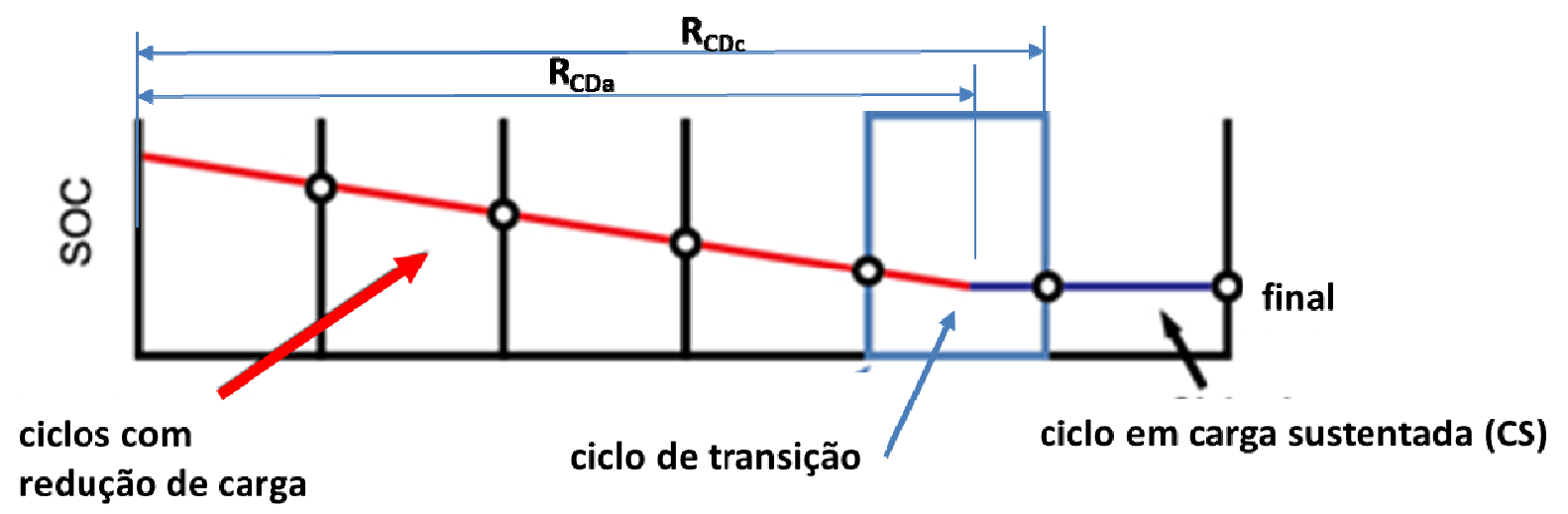

$\mathbf{R}_{\mathrm{CDa}}=$ Raio de ação ou alcance elétrico ou distância efetiva de Redução de Carga

$\mathbf{R}_{\mathrm{CDc}}=$ Distância Teórica de Redução de Carga

Figura 5 - Sequência de ciclos de teste de um veículo plug-in

A figura 6 apresenta a curva de distribuição acumulada de frequência em função da distância percorrida diariamente, em média pela frota, isto é, a probabilidade de um veículo atingir uma determinada distância em um dia de utilização, levantada a partir de pesquisas de quilometragem anual. Considerando que a resolução necessária aos cálculos é de 1 ciclo de ensaio padrão, os cálculos serão referidos às probabilidades UF1, UF2...UFn de um veículo da frota percorrer diariamente 1, 2 ...ou n ciclos por dia. Os resultados de cada ciclo serão ponderados pelas diferenças dessas probabilidades 
$\triangle \mathrm{UF1}$.....UFn e somados, sendo a última parcela o resultado do ciclo em carga sustentada ponderado pela probabilidade final (1-UFn). No exemplo desta figura as distâncias foram padronizadas em $12 \mathrm{~km}$, correspondentes ao ciclo urbano; o 5 ciclo é o último de transição e o 6 o é o ciclo em carga sustentada, como indicado na figura 5.

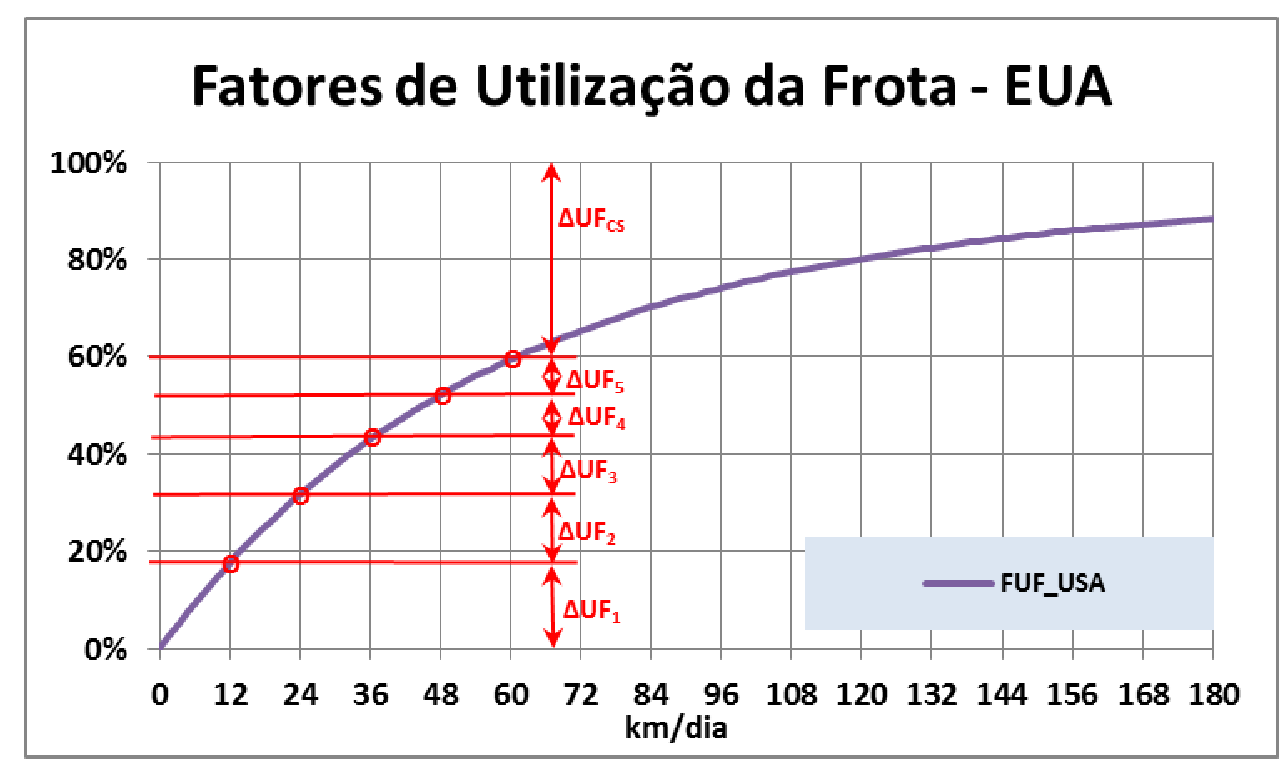

Figura 6 - Distribuição acumulada de frequência para percursos diários

Sob estes conceitos, os parâmetros de interesse são caracterizados separadamente em cada ciclo, determinado o alcance onde a energia elétrica externa atua no sistema e o número de ciclos necessários para o veículo atingir esta condição estabilizada. Assumindo-se que os ciclos tenham sido realizados dentro da distância nominal, sem violações, os resultados finais considerando todos os ciclos em redução de carga e com carga estabilizada são então computados como segue:

$$
\begin{aligned}
Y_{U F W}(g / k m) & =\sum_{1}^{n}\left(\Delta U F_{i} * Y_{i}\right)+\left(1-U F_{n}\right) * Y_{C S} \\
Y_{U F W}(g / k m) & =U F_{1} * Y_{1}+\Delta U F_{2} * Y_{2}+\cdots+\Delta U F_{n} * Y_{n}+\left(1-U F_{n}\right) * Y_{C S} \\
Y_{U F W}(g / k m) & =U F_{1} * Y_{1}+\left(U F_{2}-U F_{1}\right) * Y_{2}+\cdots+\left(U F_{n}-U F_{n-1}\right) * Y_{n}+\left(1-U F_{n}\right) * Y_{C S} \text { ou } \\
Y_{U F W}(g / k m) & =\sum_{1}^{n}\left[\left(U F_{i}-U F_{i-1}\right) * Y_{i}\right]+\left(1-U F_{n}\right) * Y_{C S}
\end{aligned}
$$

onde:

$i \quad=$ número de ciclos considerados para cada valor médio acumulado;

$n \quad=$ último ciclo ainda considerado no regime de descarga da bateria;

$Y_{i} \quad=$ massa de poluente, ou o volume de combustível, ou o consumo de energia AC, medidos em cada ciclo em gramas ou litros ou Wh/km. Para $i>n$, utilizar $Y_{i}=Y_{C S}$; no caso de energia elétrica $E_{i}=E_{C S}=0$

$Y_{U F W}(\mathrm{~g} / \mathrm{km})=$ massa de poluente, ou o volume de combustível, ou o consumo de energia $A C$, ponderados pelos fatores de utilização do sistema elétrico em cada ciclo; 


$$
\begin{array}{ll}
U F_{i} & =\text { Fator de utilizaçãodo sistema elétrico até o “io" ciclo; } \\
\Delta U F_{i} & =\text { Fator de utilização do sistema elétrico, tomado parcialmentepara cada } \\
& \text { ciclo; } \\
Y_{C S} & =\text { massa de poluente, ou o volume de combustível, ou o consumo de } \\
& \text { energia AC, medidos em regime de carga sustentada imediatamente } \\
& \text { após a estabilização da bateria em gramas ou litros ou } \mathrm{Wh} / \mathrm{km} ;
\end{array}
$$

Para melhor exatidão ou quando há violações do ciclo, recomenda-se utilizar as grandezas $Y_{i}$ acumuladas durante cada ciclo, expressas em massa, volume ou Wh, especialmente quando as distâncias percorridas em cada ciclo apresentarem diferenças significativasdevidas a violações. Neste caso deve-se multiplicar o resultado de cada ciclo pela respectiva distância " $\mathrm{D}_{\mathrm{i}}$ " e dividir pela distância total percorrida em redução de carga $\left(\mathrm{R}_{\mathrm{CDc}}=\Sigma \mathrm{D}_{\mathrm{i}}\right)$ :

$$
\begin{aligned}
& Y_{U F W}(\mathrm{~g} / \mathrm{km})=\frac{\sum_{1}^{n}\left(\Delta U F_{i} * Y_{i} * D_{i}\right)}{\sum_{1}^{n} D_{i}}+\left(1-U F_{n}\right) * Y_{C S} \\
& Y_{U F W}(\mathrm{~g} / \mathrm{km})=\frac{U F_{1} * Y_{1} * D_{1}+\left(U F_{2}-U F_{1}\right) * Y_{2} * D_{2}+\cdots+\left(U F_{n}-U F_{n-1}\right) * Y_{n} * D_{n}}{R_{C D_{C}}}+\left(1-U F_{n}\right) * Y_{C S}
\end{aligned}
$$

onde:

$D_{i} \quad=$ Distância efetivamente percorrida em cada ciclo, em km;

$R_{C D_{c}} \quad=$ Distância percorrida até o final do último ciclo onde ocorreu a estabilização da carga da bateria, em km.

\section{Curvas de Fatores de Utilização}

A Norma SAE J2841 para apresenta as origense uma listagem completa dos Fatores de Utilizaçãodisponíveis, gerados e aprovados pelo Comitê de Padrões Técnicos para VEH.

O Levantamento de Viagens Domésticas Nacionais (NHTS) foi escolhido nos Estados Unidos para ser a base do cálculo do Fator de Utilização. Este banco de dados, organizado a cada cinco anos pelo Departamento de Transportes dos E.U.A e pela Administração Federal de Rodovias, é baseado em levantamentos de motoristas por todo o território dos Estados Unidos. Mais informações sobre o NHTS podem ser obtidas acessando o website: http://nhts.ornl.gov/.

Complementarmente, foi realizado em Atlanta outro estudo muito mais detalhado, porém limitado a poucos veículos, dado o alto custo envolvido no levantamento dos dados, que determinou as distribuições acumuladas de frequência para percursos diários, identificando as viagens realizadas em ambiente urbano e em estrada. Para isso, as anotações de voluntários pesquisados indicaram que até 25 milhas por dia, as viagens são nitidamente urbanas e os percursos superiores a 60 milhas por dia exigem velocidades maiores e são obrigatoriamente em estrada. Para o intervalo intermediário o estudo considera uma variação linear de proporções (critérios 
de lógica fuzzi) e admitiu as frações de 55/45 e 43/57 como proporções prováveis entre cidade/estrada para definir o valor de corte a ser adotado para as velocidades registradas no levantamento. Em ambos os casos as curvas de probabilidade obtidas foram muito próximas.

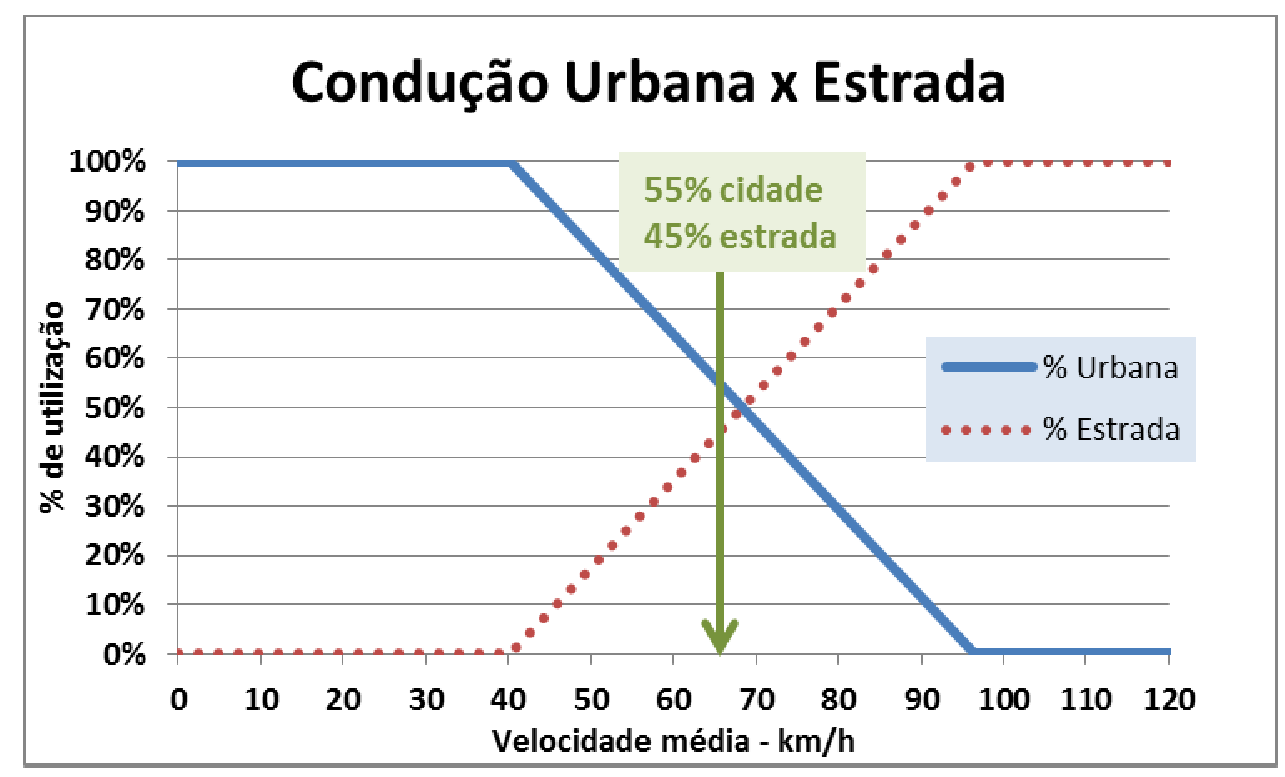

Figura 7 - Critérios para a determinação do limite de velocidade considerado para caracterizar uma viagem urbana

A comparação dos dois estudos indicou a coincidência das curvas obtidas para a frota como um todo (sem a distinção dos usos urbano e em estrada), o que justifica o primeiro método que pode abranger maior extensão do país.

Estes dois conjuntos de dados são fornecidos então como entrada para os cálculos dos Fatores de Utilização, resultando então em duas curvas que podem ser usadas, se o analista desejar determinar a utilidade da propulsão elétrica em CD para ciclos de condução urbana e em estrada separadamente. A probabilidade de um veículo percorrer uma dada distância diariamente difere um pouco do cálculo indicado na figura 4, que desconsidera a carga remanescente de um dia para outrono caso de viagens mais curtas do que o alcance com redução de carga, por este se afastar da premissa fundamental de uma recarga completa da bateria por dia.

A figura 8 apresenta estas diversas curvas, nas quais é possível perceber a diferença expressiva entre o uso urbano, em estrada e a sua composição nas proporções adotadas nas medições convencionais de consumo de combustível, bem comoindica que a consistência entre as estatísticas gerais de frota (FUF) e individuais(IUF) está preservada. 


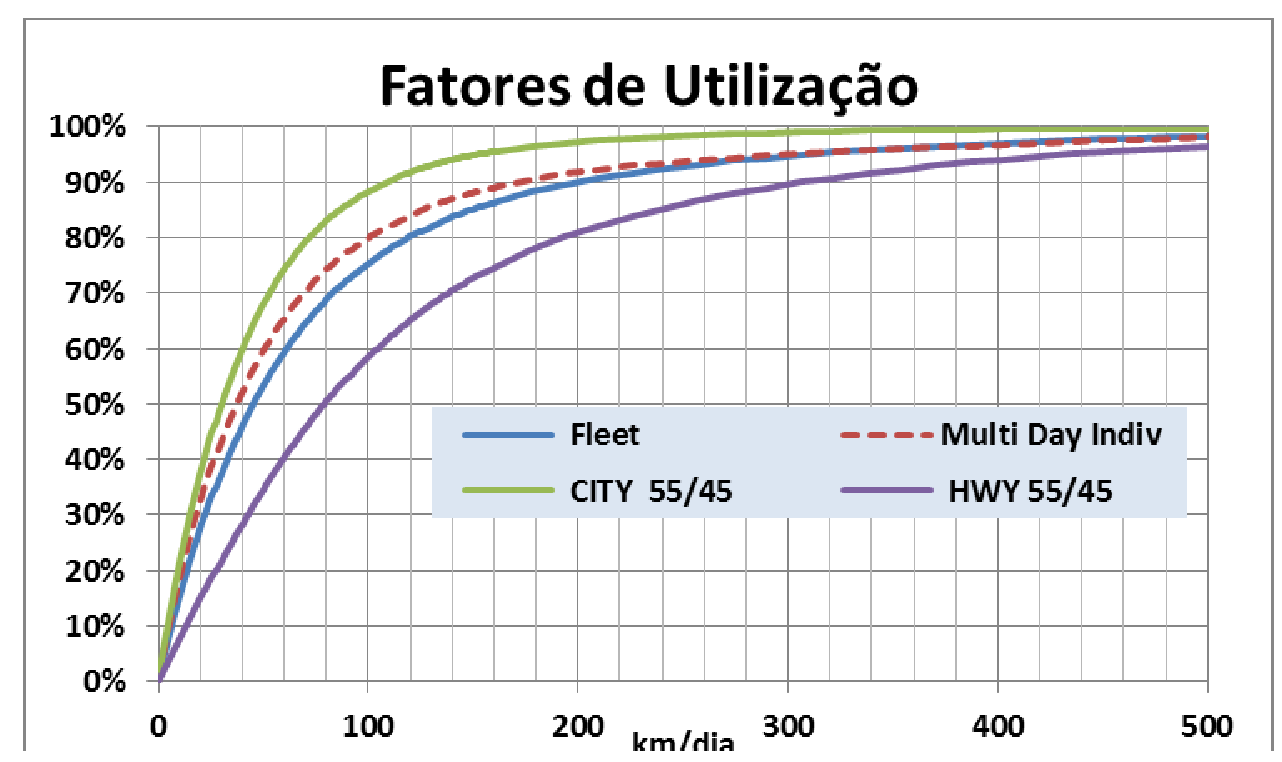

Figura 8 - Curvas do fator de utilidade da propulsão elétrica - SAE J2841

Os fatores de utilização norte-americanos foram comparados com as curvas levantadas a partir dos dados de hodômetro registrados pelo Programa de Inspeção e Manutenção na cidade de São Paulo. Para isso, a frota foi estratificada nas fases $L 4$ e L5 do PROCONVE, correspondentes aos automóveis fabricados nos períodos de 2004/2008 e 2009/2011, respectivamente, por serem de usuários com maior probabilidade de adquirirem um veículo híbrido nos próximos anos. Ressalta-se que a frota brasileira utilizada é apenas a do município de São Paulo, porém a sua quilometragem anual inclui o uso em estrada, pois as estatísticas do Programa I/M não foram idealizadas para fazerem esta distinção. Comparando-se as quilometragens anuais que apresentam a mesma probabilidade de uso, observa-se que a frota americana percorre distâncias anuais superiores às paulistanas em mais de $60 \%$ sistematicamente.

Entretanto, as curvas obtidas em São Paulo mostraram extrema coincidência com as curvas do fator de utilização da frota americana estratificada para uso urbano na proporção de $55 \%$ da quilometragem anual total, como indicado na figura 9 . Este fato demonstra que a utilização urbana brasileira é bastante semelhante à americana, porém com distâncias menores. Entretanto, é seguro admitir que o uso urbano seja muito mais intenso do que o uso em estrada no Brasil, o que sugere que a norma brasileira adote as curvas de São Paulo até que levantamentos sejam realizados com extensão nacional e demonstrem fatores diferentes dos observados na nossa realidade. Esta hipótese é particularmente válida quando se considera que os maiores volumes de combustíveis são consumidos nas regiões metropolitanas e grandes cidades. 


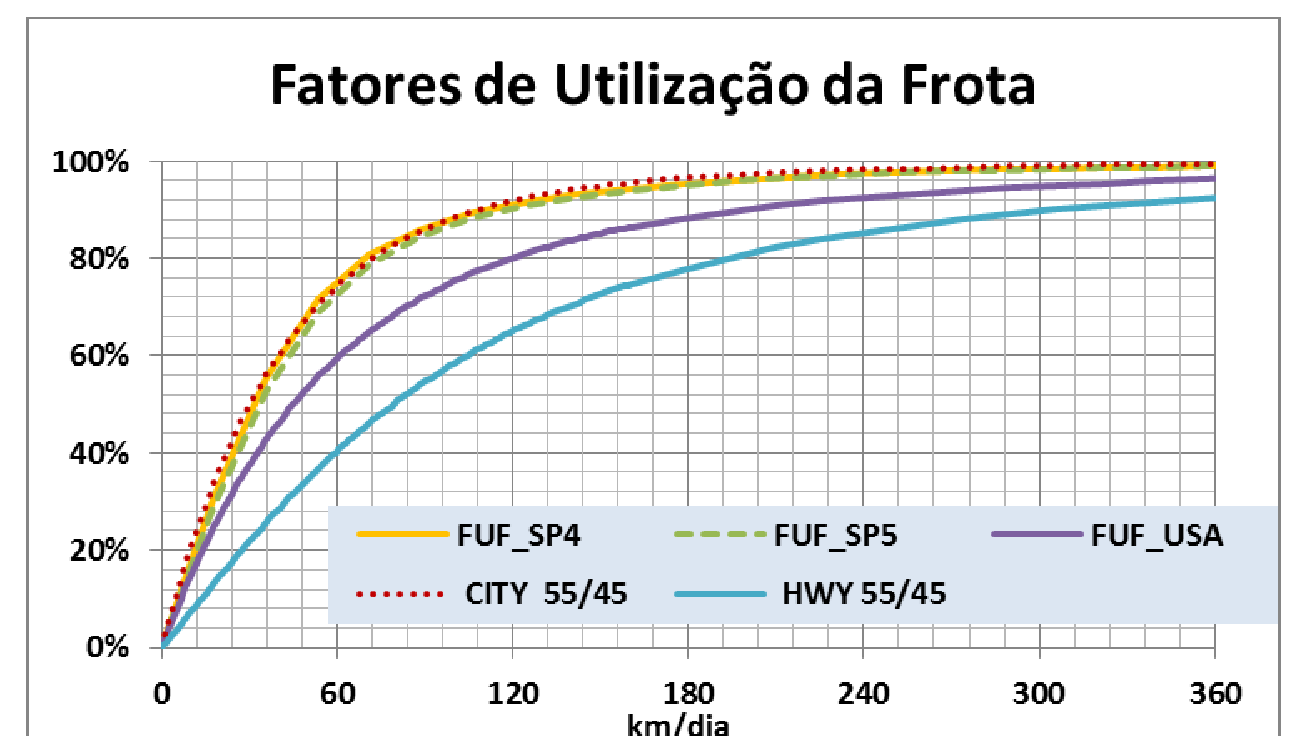

Figura 9 - Curvas do fator de utilição americanos e de São Paulo

\section{Conclusões e recomendações}

As principais conclusões deste trabalho justificam a adoção dos princípios e conceitos da norma SAE J1711 no Brasil, com três alterações conceituais para os veículos plug-in em regime de redução de carga da bateria:

a) para divulgação aos usuários, recomendar e especificar a apresentação de resultados de emissão, de consumo de combustível e de energia elétrica sob a forma de curvas das médias acumuladas destes parâmetros desde o primeiro ciclo de condução até pelo menos $200 \mathrm{~km}$ de viagens sucessivas;

b) para certificação de emissões e eficiência energética, definir dois conjuntos de resultados:

- um como a média acumulada de cada parâmetro desde o primeiro ciclo de condução até o ciclo de transição;

- outro como as média ponderadas de cada parâmetro nos ciclos urbano (com partidas a quente e a frio) e as médias no ciclo estrada, todos ensaiados em regime de carga sustentada;

c) para efeito de inventário de emissões e consumos médios de uma frota, manter os procedimentos de cálculo atualmente definidos, porém com a curva dos Fatores de Utilização obtida em frotas brasileiras de veículos. atualmente existe somente os dados de São Paulo, mas estas curvas devem ser revistas periodicamente. 


\section{Bibliografia}

SAE J1711 - Recommended Practice for Measuring the Exhaust Emissionsand Fuel Economy of Hybrid-Electric Vehicles, Including Plug-in Hybrid Vehicles - Jun 2010

SAE J2841 - Utility Factor Definitions for Plug-In Hybrid Electric Vehicles Using Travel Survey Data - Sept 2010

http://nhts.ornl.gov/

Duoba, M. etalii - PHEV Testing - SAE J1711-revision - Argonne National LaboratoryAdvanced Powertrain Research Facility - 2008

Duoba,M. etalii- Argonne Facilitation of PHEV Standard Testing Procedure (SAE J1711) - Argonne National Laboratory - Advanced Powertrain Research Facility - 2006

Gonder, J. andSimpson, A. - Measuring and Reporting Fuel Economy of Plug-In Hybrid Electric Vehicles_NREL Report 2006

http://www.rita.dot.gov/bts/sites/rita.dot.gov.bts/files/publications/national_transportation statistics/index.html 